\title{
Impact of diabetes-associated lipoproteins on oxygen consumption and mitochondrial enzymes in porcine aortic endothelial cells ${ }^{\star}$
}

\author{
Xueping Xie, Subir Roy Chowdhury, Ganesh Sangle and Garry X. Shen ${ }^{\varpi}$ \\ Departments of Internal Medicine and Physiology, University of Manitoba, Canada
}

\begin{abstract}
Impairments in mitochondrial function have been proposed to play an important role in the pathogenesis of diabetes. Atherosclerotic coronary artery disease (CAD) is the leading cause of mortality in diabetic patients. Mitochondrial dysfunction and increased production of reactive oxygen species (ROS) are associated with diabetes and CAD. Elevated levels of glycated low density lipoproteins (glyLDL) and oxidized LDL (oxLDL) were detected in patients with diabetes. Our previous studies demonstrated that oxLDL and glyLDL increased the generation of ROS and altered the activities of antioxidant enzymes in vascular endothelial cells (EC). The present study examined the effects of glyLDL and oxLDL on mitochondrial respiration, membrane potential and the activities and proteins of key enzymes in mitochondrial electron transport chain (mETC) in cultured porcine aortic EC (PAEC). The results demonstrated that glyLDL or oxLDL significantly reduced oxygen consumption in Complex I, II/III and IV of mETC in PAEC compared to LDL or vehicle control using oxygraphy. Incubation with glyLDL or oxLDL significantly reduced mitochondrial membrane potential, the activities of mitochondrial ETC enzymes - NADH dehydrogenase (Complex I), succinate cytochrome $c$ reductase (Complex II + III), ubiquinol cytochrome $c$ reductase (Complex III), and cytochrome $c$ oxidase (Complex IV) in PAEC compared to LDL or control. Treatment with oxLDL or glyLDL reduced the abundance of subunits of Complex I, ND1 and ND6 in PAEC. However, the effects of oxLDL on mitochondrial activity and proteins were not significantly different from glyLDL. The findings suggest that the glyLDL or oxLDL impairs mitochondrial respiration, as a result from the reduction of the abundance of several key enzymes in mitochondria of vascular EC, which potentially may lead to oxidative stress in vascular EC, and the development of diabetic vascular complications.
\end{abstract}

Key words: low density lipoprotein, respiration chain, mitochondrial oxygen, consumption, mitochondrial membrane potential, vascular endothelial cells

Received: 18 August, 2010; accepted: 09 October 2010; available on-line: 27 October, 2010

\section{INTRODUCTION}

Hyperglycemia and dyslipidemia are two major biochemical markers of diabetes. Cardiovascular diseases are the predominant cause of death in diabetic patients. The most common cardiovascular complication in diabetic patients is atherosclerotic coronary artery disease (Bartnik et al., 2007). Elevated low density lipoprotein (LDL) is a classical risk factor for atherosclerotic cardiovascular disease. Increased levels of oxidized LDL (oxLDL) and glycated LDL (glyLDL) have been detected in diabetic patients (Lyons, 1993; Tsimikas et al., 2003). Our previous studies demonstrated that oxLDL and glyLDL increased the generation of reactive oxygen species (ROS) in vascular endothelial cells (EC) (Zhao \& Shen, 2005). ROS is implicated in endothelial dysfunction and diabetic vascular complications. Mitochondria are an important source of ROS in cells. We hypothesize that oxLDL or glyLDL may impair mitochondrial respiratory function in vasculature. Endothelium is a single layer cellular barrier between blood components and vascular wall. Interactions between modified LDL and vascular EC play important roles in the pathophysiology of atherosclerosis and thrombosis. Our group recently demonstrated that oxLDL or glyLDL impaired mitochondrial electron transport chain (mETC) complexes activity in EC (Roy Chowdhury et al., 2010; Sangle et al., 2010). The effects of oxLDL and glyLDL on mETC activity in EC have not been compared in parallel. The impact of glyLDL or oxLDL on the proteins of the key enzymes in mETC in EC has not been documented. The present study compared the effects of oxLDL and glyLDL on mitochondrial activities and examined the impact of diabetes-associated LDLs on the abundance of key enzymes in mitochondrial ETC in cultured porcine aortic EC (PAEC).

\section{MATERIALS AND METHODS}

Isolation and modification of lipoproteins. LDL (density 1.019-1.063) was isolated from plasma of healthy donors using sequential density floatation ultracentrifugation. LDL was oxidized through dialysis against $5 \mu \mathrm{M} \mathrm{CuSO}$ for $24 \mathrm{~h}$ at $22^{\circ} \mathrm{C}$ (Ren et al., 1997). The oxidation of LDL was verified using thiobarbituric acid reactive substance assay and non-denatured gel electrophoresis. GlyLDL was prepared by incubation of LDL with $50 \mathrm{mM}$ glucose and $50 \mathrm{mM}$ sodium cyanoborohy-

e-mail: gshen@ms.umanitoba.ca

* This work was presented in the poster form at the 16th European Bioenergetics Conference (Warsaw, 2010); abstract in Biochim Biophys Acta, 1797 (Suppl): 53-54 (2010).

Abbreviations: CS, citrate synthase; EC, endothelial cells; glyLDL, glycated LDL; LDL, low density lipoprotein; mETC, mitochondrial electron transport chain; ND, NADH dehydrogenase; oxLDL, oxidized LDL; PAEC, porcine aortic endothelial cells; ROS, reactive oxygen species; SCCR, succinate cytochrome $c$ reductase; TMRM, tetramethylrhodamine metyl ester 
dride in the presence of $0.01 \%$ EDTA for 2 weeks at $37^{\circ} \mathrm{C}$ as previously described (Zhang et al., 1998). Free glucose or chemicals in glyLDL preparation was removed via dialysis. Approximately $60 \%$ of lysine residues were glycated in the preparations of glyLDL used in following experiments assessed using trinitrobenzenesulfonic acid assay. The level of endotoxin in lipoproteins was monitored using E-Toxate kit with a threshold of 0.05 $\mathrm{ng} / \mathrm{ml}$ (Sigma, St. Louis, MO, USA). Lipoproteins were stored in sealed tubes under a layer of nitrogen at $4{ }^{\circ} \mathrm{C}$ in dark to prevent auto-oxidation.

Cell culture and experimental incubation. PAEC were obtained from Dr. P. E. DiCorleto at the Cleveland Clinic Foundation (Shen et al., 1989). PAEC were grown in Dulbecco's modified Eagle medium (Invitrogen, ON, Canada) supplemented with 10\% fetal bovine serum and $1 \%$ penicillin-streptomycin (Invitrogen). Cells were cultured in an incubator at $37^{\circ} \mathrm{C}$ and maintained in a humidified atmosphere containing $5 \% \mathrm{CO}_{2}$. PAEC were treated with vehicle, lipoproteins at indicated concentration and time at $37^{\circ} \mathrm{C}$ under $5 \% \mathrm{CO}_{2}$.

Measurement of mitochondrial oxygen consumption using oxygraphy. Oxygen consumption was determined at $37^{\circ} \mathrm{C}$ using OROBOROS Oxygraphy-2K (Oroboros, Innsbruck, Austria) (Chowdhury et al., 2000). PAEC were trypsinized and counted using hemocytometer. Cells were resuspended in the $\mathrm{KCl}$ medium (80 mM KCl, $10 \mathrm{mM}$ Tris/ $\mathrm{HCl}, 3 \mathrm{mM} \mathrm{MgCl}$, $1 \mathrm{mM}$ EDTA, $5 \mathrm{mM}$ potassium phosphate, $\mathrm{pH}$ 7.4) to $1.5 \times 10^{6}$ cells $/ \mathrm{ml}$. To permeabilize plasma membrane for the access of substrates to mitochondria, EC were treated with digitonin $\left(25 \mu \mathrm{g} / 2 \mathrm{ml}\right.$ for $10^{6}$ cells $)$. At this concentration of digitonin, intact mitochondria in EC were maintained. Various substrates and inhibitors for mitochondrial respiratory chain complexes were added as described in Fig. 1. Unless mentioned otherwise, all the chemicals were obtained from Sigma (Sigma, MO, USA). Oroboros DatLab software was used for the calculation and graphic presentation of oxygen consumption. Oxygen consumption was normalized by cell numbers and presented as pmol $\mathrm{O}_{2} / \mathrm{s}$ per $10^{6}$ cells.

Assessment of mitochondrial membrane potential. PAEC were treated with $100 \mu \mathrm{g} / \mathrm{ml}$ of oxLDL or glyLDL for $12 \mathrm{~h}$. After treatment, cells were harvested by trypsinisation, washed three times in cold phosphatebuffered saline, counted and resuspended in the $\mathrm{KCl}$ at a protein concentration of $1 \mathrm{mg} / \mathrm{ml}$. Digitonin was added at standardized concentration (0.02 mg digitonin $/ \mathrm{mg}$ protein), cells were incubated for $5 \mathrm{~min}$ on ice and spin for $5 \mathrm{~min}$ at $600 \times \mathrm{g}$. The supernatant was removed after centrifugation and cells were resuspended in $0.5 \mathrm{ml} \mathrm{KCl}$ medium. The permeabilised cells were incubated with 20 $\mathrm{nM}$ tetramethylrhodamine methyl ester (TMRM), a mitochondrial membrane potential probe (Molecular Probes, Eugene, OR, USA), for $10 \mathrm{~min}$ at room temperature. Mitochondrial membrane potential $\left(\Delta \Psi_{\mathrm{m}}\right)$ measurements were performed in the presence of $10 \mathrm{mM}$ succinate and $1 \mu \mathrm{M}$ rotenone using MoFloXDP Coulter flow cytometer (Beckman) equipped with an argon laser 488 nm (Floryk \& Houstěk, 1999). TMRM signal was analyzed in the FL2 channel, equipped with band pass filter $575 \pm 25 \mathrm{~nm}$. Approximately 23000 cells were used for each measurement. Data were acquired and analyzed in $\log$ scale using Summit 5.2 software (Beckman). Arithmetic mean values of TMRM fluorescence signal in arbitrary units were calculated for each condition for graphic representation.
NADH dehydrogenase (ND, Complex I) activity. ND activity was measured as described previously (Birch-Machin et al., 1994). Mitochondrial fraction of PAEC $(50 \mu \mathrm{g})$ was added to a buffer containing $25 \mathrm{mM}$ potassium phosphate ( $\mathrm{pH} 7.2), 5 \mathrm{mM} \mathrm{MgCl}_{2}, 2 \mathrm{mM}$ $\mathrm{KCN}, 2.5 \mathrm{mg} / \mathrm{ml}$ bovine serum albumin (fraction $\mathrm{V}$ ), $2 \mu \mathrm{g} / \mathrm{ml}$ antimycin A, $0.1 \mathrm{mM} \mathrm{NADH}$, and $50 \mu \mathrm{M}$ decylubiquinone. The measurement of Complex I activity was started at $3 \mathrm{~min}$ before the addition of rotenone $(2 \mu \mathrm{g} / \mathrm{ml})$ and continued for another $3 \mathrm{~min}$ at $340 \mathrm{~nm}$ using an Ultrospec 2000 UV-visible spectrophotometer equipped with Biochrom Swift II software (Biopharmacia Biotech, Uppsala, Sweden).

Succinate cytochrome $c$ reductase (SCCR, Complex II/III) activity. SCCR activity was measured by monitoring the rate of reduced cytochrome $c$ formation using succinate as substrate. The reaction mixture contained $10 \mathrm{mM}$ potassium phosphate ( $\mathrm{pH} 7.4$ ), $2 \mathrm{mM}$ EDTA, $0.01 \%$ bovine serum albumin (fatty acid free), $0.2 \mathrm{mM}$ ATP, $1 \mathrm{mM}$ $\mathrm{KCN}, 5 \mu \mathrm{M}$ rotenone, and $10 \mathrm{mM}$ succinate (Chowdhury et al., 2005). Sonicated total cellular proteins ( $0.2 \mathrm{mg}$ protein) were incubated with the reaction mixture for $3 \mathrm{~min}$ and the reaction was started by the addition of $40 \mu \mathrm{M}$ oxidized cytochrome $c$. Changes in absorbance were monitored at $30^{\circ} \mathrm{C}$ using a spectrophotometer for $5 \mathrm{~min}$ at $550 \mathrm{~nm}$.

Ubiquinol cytochrome $c$ reductase (UCCR, Complex III) activity. UCCR activity was evaluated using $100 \mu \mathrm{g}$ of cell lysates with a reaction mixture containing $25 \mathrm{mM}$ potassium phosphate ( $\mathrm{pH} 7.4), 5 \mathrm{mM} \mathrm{MgCl}_{2}$, $2 \mathrm{mM} \mathrm{KCN}, 2 \mu \mathrm{g} / \mathrm{ml}$ rotenone, $2.5 \mathrm{mg} / \mathrm{ml}$ bovine serum albumin, and $50 \mu \mathrm{M}$ cytochrome $c$ in a final volume of $1 \mathrm{ml}$. After a 2-min equilibration period, the reaction was started by the addition of $50 \mu \mathrm{M}$ ubiquinol- 2 and the increase in absorbance at $550 \mathrm{~nm}$ was monitored using a spectrophotometer (Vondra et al., 1977).

Cytochrome $c$ oxidase (COX, Complex IV) activity. COX activity was examined at $30^{\circ} \mathrm{C}$ by following the rate of oxidation of reduced cytochrome $c$ at $550 \mathrm{~nm}$. The assay in cultured cells was performed in the presence of $40 \mu \mathrm{M}$ reduced cytochrome c, $20 \mathrm{mM}$ phosphate buffer, $0.1 \mathrm{mg}$ of protein from cultured cells, and $16 \mathrm{mg}$ of lauryl maltoside/mg protein (0.16\%) (Chowdhury et al. 2007).

Citrate synthase (CS) activity. CS activity was determined at $30^{\circ} \mathrm{C}$ in a medium containing $150 \mathrm{mM}$ Tris $/ \mathrm{HCl}$ ( $\mathrm{pH} 8.2$ ), $0.16 \%$ of lauryl maltoside, $0.1 \mathrm{mM}$ dithionitrobenzoic acid, and $0.1 \mathrm{mg}$ protein from PAEC. The reaction was started by the addition of $300 \mu \mathrm{M}$ acetyl-CoA, and changes in absorbance at $412 \mathrm{~nm}$ were measured for $1 \mathrm{~min}$. This rate was subtracted from that with the addition of $0.5 \mathrm{mM}$ oxalacetic acid. CS activity was used to determine the amount of functional mitochondria in cells (Srere, 1969).

Western blotting. Western blotting analysis was performed as previously described (Sangle et al., 2010). Equal amounts of total cellular proteins were run on $12 \%$ SDS/PAGE and electrotransferred to nitrocellulose membrane using antibodies against subunits of $\mathrm{NADH}$ dehydrogenase (ND1, ND6) (Santa Cruz, CA, USA) or porin (Abcam, Cambridge, MA, USA). Enhanced chemiluminescence reagents (Amersham, Piscataway, NJ, USA) were used for detecting targeted antigens on membrane. The densities of antigens were analyzed using ChemiDoc system with Quantity-One software (Bio-Rad, Hercules, CA, USA). The abundance of targeted proteins on membrane was normalized with the level of porin, a control protein in mitochondria.

Statistical analysis. The data were presented as means of three replicates \pm standard deviation (S.D.). All 
results were processed by the one-way variance analysis (ANOVA). Differences at $P<0.05$ were considered as significant.

\section{RESULTS}

\section{Effects of oxLDL and glyLDL on mitochondrial oxygen consumption}

The oxygen consumption of mitochondrial complexes in digitonin-permeabilized PAEC was examined using oxygraphy with complex-specific substrates and inhibitors. The oxygen consumption of Complex I was determined as rotenone-sensitive respiration in the presence of NADH-

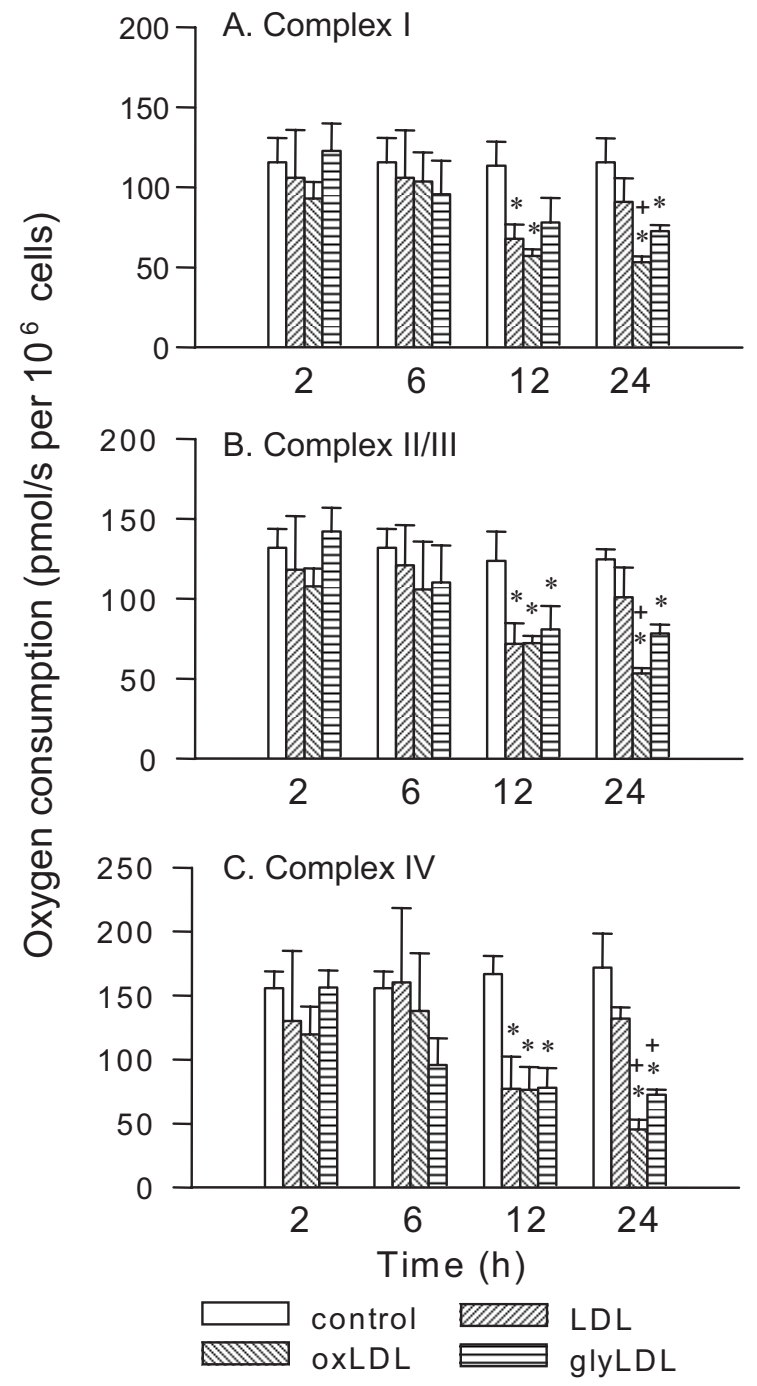

Figure 1. Effect of oxLDL and glyLDL on oxygen consumption in porcine aortic endothelial cells (PAEC)

Confluent PAEC were treated with $100 \mu \mathrm{g} / \mathrm{ml}$ of $\mathrm{LDL}$, oxLDL or glyLDL for $24 \mathrm{~h}$. Cells were trypsinized and counted. Cell suspension $\left(3 \times 10^{6}\right.$ cells) in the $\mathrm{KCl}$ buffer was added in a chamber of oxygraphy. Oxygen concentration (left axis) and oxygen consumption rate or slope (right axis) were instantly analyzed. (A) Complex I oxygen consumption was assessed from rotenone-sensitive oxygen consumption induced by glutamate + malate. (B) Complex II/III activity was assessed from succinate-induced oxygen consumption. (C) TMPD-induced oxygen consumption. Values were presented in mean \pm S.D. $(n=3)$ after justification with cell numbers. ${ }^{*} P<0.05$ versus control; $+P<0.05$ versus $\mathrm{LDL}$. dependent substrates. The consumption of Complexes II and III were evaluated as succinate-antimycin A-sensitive respiration in the presence of flavoprotein-dependent substrates. The respiratory capacity of Complex IV was evaluated in the presence of ascorbate and $N, N, N^{\prime}, N^{\prime}$ tetramethyl-p-phenylendiamine dihydrochloride (TMPD). Our previous studies demonstrated that $100 \mu \mathrm{g} / \mathrm{ml}$ of oxLDL or glyLDL for $<24 \mathrm{~h}$ induced increase of ROS production without detectable cellular injury of PAEC (Zhao \& Shen, 2005). PAEC were treated with $100 \mu \mathrm{g} / \mathrm{ml}$ of LDL, oxLDL of glyLDL for 2-24 h. OxLDL or glyLDL significantly reduced oxygen consumption in Complex I, II + III or IV in EC after 12-24 h of incubation compared to control $(P<0.05$, Fig. 1A-C). LDL treatment moderately, but not significantly, reduced oxygen consumption in Complex I, II+III or IV. Treatment with oxLDL or glyLDL for $24 \mathrm{~h}$ induced significant decreases in oxygen consumption in Complex I, II+III or IV compared to LDL $(P<0.05$, Fig. 1A-C).

\section{Effects of oxLDL and glyLDL on mitochondrial membrane potential}

Our earlier study demonstrated that glyLDL reduced mitochondrial membrane potential in EC (Sangle et al., 2010). The present study compared the effects of oxLDL and glyLDL on membrane potential in PAEC. Fig. 2 demonstrated that the effect of oxLDL on mitochondrial membrane potential was comparable to that of glyLDL. Both types of diabetes-associated lipoproteins significantly reduced mitochondrial membrane potential in PAEC $(P<0.01$, Fig. 2).

\section{Effects of oxLDL and glyLDL on enzymatic activity of $\mathrm{mETC}$}

Treatment with $100 \mu \mathrm{g} / \mathrm{ml}$ of oxLDL, glyLDL or LDL for $12 \mathrm{~h}$ significantly decreased the enzymatic activity of Complex I in PAEC compared to control (Fig. 3A). OxLDL or glyLDL induced significantly greater decrease in Complex I activity compared to LDL $(P<0.05$, Fig. 3A). OxLDL, glyLDL or LDL significantly reduced SCCR activity compared to control $(P<0.05)$. OxLDL, but not glyLDL, induced significantly greater inhibition on SCCR activity compared to LDL $(P<0.05$, Fig. 3B). OxLDL,

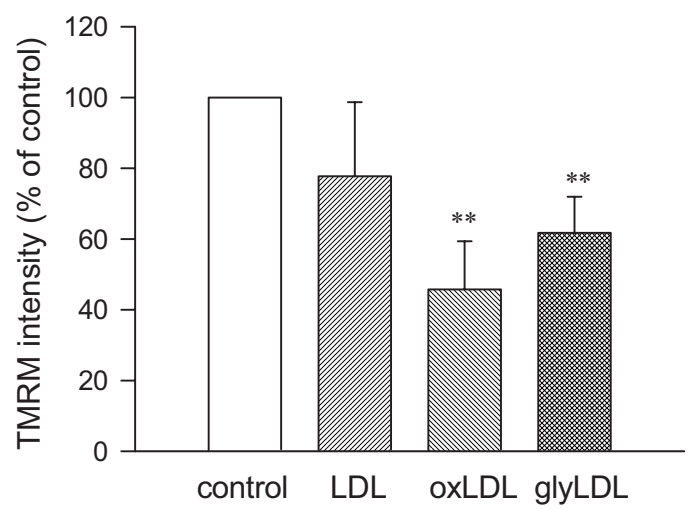

Figure 2. Effects of oxLDL and glyLDL on mitochondrial membrane potential in PAEC

PAEC cultures were treated with vehicle (control), $100 \mu \mathrm{g} / \mathrm{ml}$ of oxLDL or glyLDL for $12 \mathrm{~h}$. Cells were harvested and treated with $0.02 \mathrm{mg} / \mathrm{mg}$ protein of digitonin, then incubated with TMRM. Cytofluorimetric analysis was undertaken using flow cytometer. Changes in TMRM intensity were expressed in mean \pm S.E. in percent of control $\left(\mathrm{n}=3\right.$ experiments). ${ }^{*} P<0.01$ versus control. 

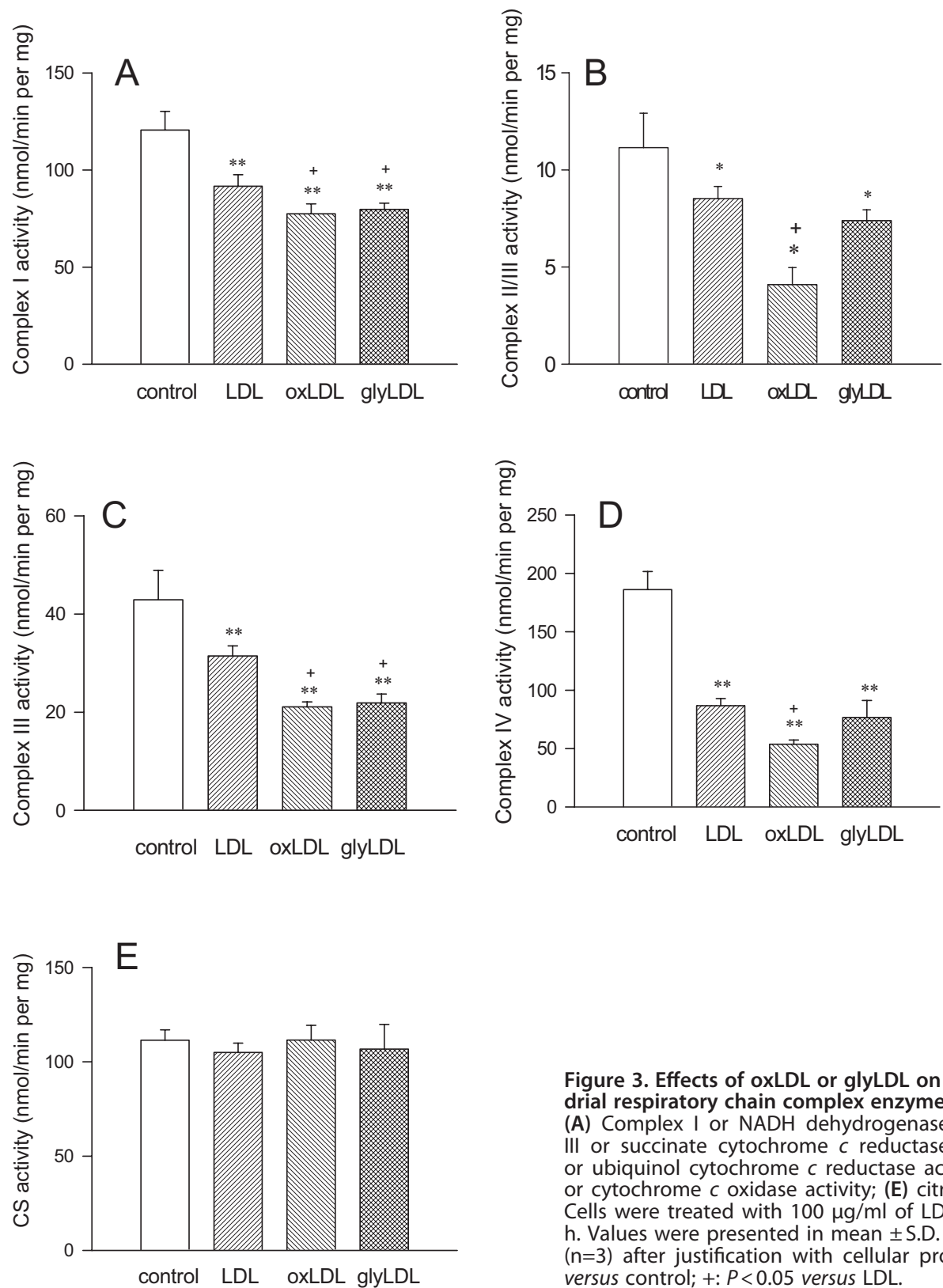

glyLDL or LDL significantly reduced Complex III activity compared to control $(P<0.01)$. The reductions in Complex III activity in PAEC exposed to oxLDL or glyLDL were significantly greater compared to LDL $(P<0.05$, Fig. 3C). OxLDL, glyLDL or LDL treatment significantly deceased the activity of Complex IV by $>50 \%(P<0.01)$. OxLDL, but not glyLDL, induced greater decrease in the activity of cytochrome $c$ oxidase compared to LDL $(P<0.05$, Fig. 3D). In comparison, LDL or its modified forms did not significantly alter the activity of CS (Fig. 3E), which is a mitochondrial matrix-soluble enzyme and relatively insensitive to the effects of oxidants (Masaki et al., 1995).

\section{Effects of oxLDL and glyLDL on abundance of subunits of NADH-dehydrogenase, ND1 and ND6}

To address the question whether oxLDL or glyLDL reduces the levels of key mETC complex enzymes, the abundances of components of Complex I, ND1 and

Figure 3. Effects of oxLDL or glyLDL on the activity of mitochondrial respiratory chain complex enzyme activities in PAEC (A) Complex I or NADH dehydrogenase activity; (B) Complex II/ III or succinate cytochrome $c$ reductase activity; (C) Complex III or ubiquinol cytochrome $c$ reductase activity and (D) Complex IV or cytochrome $c$ oxidase activity; (E) citrate synthase (CS) activity. Cells were treated with $100 \mu \mathrm{g} / \mathrm{ml}$ of LDL, oxLDL or glyLDL for 12 $\mathrm{h}$. Values were presented in mean \pm S.D. $\mathrm{nmol} / \mathrm{min}$ per $\mathrm{mg}$ protein $(n=3)$ after justification with cellular proteins. ${ }^{*}, * * P<0.05$ or 0.01 versus control; $+: P<0.05$ versus $L D L$.

ND6 were examined. The results indicated that oxLDL and glyLDL significantly decreased the abundances of ND1, and ND6 compared to vehicle control, but did not evidently alter the level of porine in PAEC (Fig. 4).

\section{DISCUSSION}

The present study demonstrated that oxLDL and glyLDL significantly inhibited oxygen consumption, membrane potential, mETC enzymatic activity and the abundances of ND1 and ND6 compared to control in cultured PAEC.

Elevated levels of oxLDL and glyLDL are detected in diabetic patients (Lyons, 1993; Tsimikas et al., 2003). Previous studies demonstrated that hyperglycemia increased the oxidation of LDL (Lyons, 1993). Our group previously reported that increased lipid peroxides were detected in glyLDL. Prolonged incubation with EC increased 

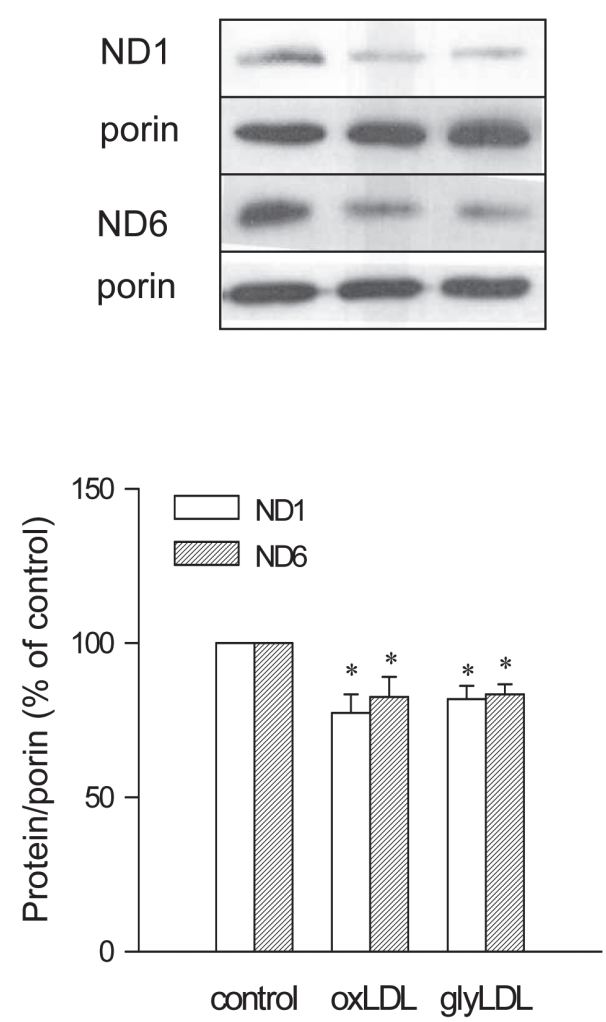

Figure 4. Effects of oxLDL and glyLDL on the abundance of mitochondrial proteins

PAEC were incubated with $100 \mu \mathrm{g} / \mathrm{ml}$ of oxLDL, glyLDL or vehicle (control) for $12 \mathrm{~h}$. Cellular proteins were analyzed using Western blotting with antibody for NADH dehydrogenase 1 and 6 (ND1, ND6) or porin. The levels of ND1 or ND6 were normalized with porin in corresponding lanes. Values were expressed in mean \pm S.D. $\%$ of control ( $n=3$ experiments). ${ }^{*} P<0.05$ versus control.

lipid peroxidation in glyLDL. Antioxidants reduced the formation of lipid peroxides in LDL or glyLDL (Ren et al., 2000). The present study compared the effects of oxLDL and glyLDL on mitochondrial oxygen consumption and enzymatic activities of mETC Complex I-IV in PAEC in parallel. The results indicated that both oxLDL and glyLDL decreased mitochondrial oxygen consumption and mETC enzymes activities. The findings suggest that both oxLDL and glyLDL impair mitochondrial respiration. Persistent hyperglycemia and hypercholesterolemia may contribute to the deterioration of mitochondrial activity in diabetic patients.

Complex I and III have been considered as the major sources of ROS in mETC. Previous studies demonstrated that hyperglycemia and diabetic conditions may reduce Complex III activity (Kowluru et al., 2006; Munusamy et al., 2009). Ceaser et al. (2003) found that nonapoptic oxLDL increased Complex I enzyme activity in EC. Earlier study in our laboratory demonstrated that extensively oxLDL, in an extent of oxidation similar as that used in the present study, reduced Complex I activity compared to control of LDL, which suggest the inhibition of oxLDL on Complex I activity may depend on the extensity of oxidation in LDL. In the presence of uncoupler, ADP-induced oxygen consumption increased by $50 \%$, which suggests a large portion of oxLDL-induced decrease in mitochondrial respiration is not due to the reduction of ATP synthesis in PAEC (Roy Chowdhury et al., 2010). The present study demonstrated that oxLDL reduced the activities of Complex I, II/III, III and IV compared to vehicle or LDL. GlyLDL treatment induced significant decreases in activities of enzymes in Complex I-IV compared to control, but only had significant reduction in Complex I and III activities compared to LDL in PAEC. The results of the present study are consistent to our earlier findings that both oxLDL and glyLDL increased the generation of ROS from EC (Zhao \& Shen, 2005) and also supports previous findings of the reduction of Complex III activity in diabetes (Kowluru et al., 2006; Munusamy et al., 2009).

ND complex contains more than 40 subunits. Seven of Complex I subunits, including ND1 and ND6, are encoded by mitochondrial DNA (mtDNA) (DiMauro \& Andreu, 2000). Unlike nuclear DNA, mtDNA lacks of the protection of histone that might contribute to enhanced susceptibility of mtDNA to oxidative damage. Proximity of mtDNA to ROS production site in mitochondria is a possible risk for high rate of mtDNA mutation. In addition, mtDNA attaches to inner membrane and close to ROS in matrix generated from mitochondrial respiration. Mitochondrial enzyme subunits encoded by mtDNA (including ND1-6) are expected to be more susceptible to oxidative stress than nuclear DNA encoded mETC subunits. The present study demonstrated that abundances of ND1, and ND6 proteins were significantly reduced by oxLDL or glyLDL. The results suggest that the reduction of Complex I activities induced by oxLDL or glyLDL may result from the decreases in the levels of mtDNA encoded Complex I enzyme subunits, including, but not limited to, ND1 and ND6, in PAEC. Relationship between the diabetes-associated LDLs and subunits in other mtDNA encoded mETC enzymes remains to be investigated in subsequent studies. OxLDL or glyLDL contains lipid peroxidative products, which may impair mitochondrial respiration. Increased ROS production via mitochondrial respiration may damage $\mathrm{mtDNA}$ and reduce the content of mtDNA encoded mETC enzymes in PAEC exposed to oxLDL or glyLDL.

In conclusion, the present study for the first time directly compared the effects of oxLDL and glyLDL on mitochondrial oxygen consumption, membrane potential and enzymatic activities in EC, and originally demonstrated that the diabetes-associated lipoproteins reduced the abundances of two subunits of mETC Complex I enzyme, ND1 and ND6, in PAEC. These changes in mitochondrial activities may contribute to the development of cardiovascular complications in diabetic patients.

\section{Acknowledgements}

The authors thanks for the grant supports from the Heart and Stroke Foundation, the Canadian Institutes of Health Research and the Canadian Diabetes Association to G. X. Shen. G. Sangle was a Studentship Award from the Manitoba Health Research Council.

\section{REFERENCES}

Bartnik M, Norhammar A, Rydén L (2007) Hyperglycaemia and cardiovascular disease. I Intern Med 262: 145-156.

Birch-Machin MA, Briggs HL, Saborido AA, Bindoff LA, Turnbull DM (1994) An evaluation of the measurement of the activities of complexes I-IV in the respiratory chain of human skeletal muscle mitochondria. Biochem Med Metab Biol 51: 35-42.

Ceaser EK, Ramachandran A, Levonen AL, Darley-Usmar VM (2003) Oxidized low-density lipoprotein and 15 -deoxy- $\Delta^{12,14}-$ PGJ2 increase mitochondrial complex I activity in endothelial cells. Am J Physiol Heart Circ Physiol 285: H2298-H2308. 
Chowdhury SK, Drahota Z, Floryk D, Calda P, Houstek J (2000) Activities of mitochondrial oxidative phosphorylation enzymes in cultured amniocytes. Clin Chim Acta 298: 157-173.

Chowdhury SK, Gemin A, Singh G (2005) High activity of mitochondrial glycerophosphate dehydrogenase and glycerophosphate-dependent ROS generation in prostate cancer cell lines. Biochem Biophys Res Commun 333: 1139-1145.

Chowdhury SK, Raha S, Tarnopolsky MA, Singh G (2007) Increased expression of mitochondrial glycerophosphate dehydrogenase and antioxidant enzymes in prostate cancer cell lines/cancer. Free Radic Res 41: 1116-1124.

DiMauro S, Andreu AL (2000) Mutations in mtDNA: are we scraping the bottom of the barrel? Brain Pathol 10: 431-441.

Floryk D, Houstěk J (1999) Tetramethyl rhodamine methyl ester (TMRM) is suitable for cytofluorometric measurements of mitochondrial membrane potential in cells treated with digitonin. Biosci Rep 19: 27-34.

Kirkland RA, Franklin JL (2001) Evidence for redox regulation of cytochrome $c$ release during programmed neuronal death: antioxidant effects of protein synthesis and caspase inhibition. $J$ Neuroscience 21: 1949-1963.

Kowluru RA, Kowluru V, Xiong Y, Ho YS (2006) Overexpression of mitochondrial superoxide dismutase in mice protects the retina from diabetes-induced oxidative stress. Free Radic Biol Med 41: 1191-1196.

Lyons TJ (1993) Glycation and oxidation: a role in the pathogenesis of atherosclerosis. Am J Cardiol 71: 26B-31B.

Masaki H, Atsumi T, Sakurai H (1995) Detection of hydrogen peroxide and hydroxyl radicals in murine skin fibroblasts under UVB irradiation. Biochem Biophys Res Commun 206: 474-479.

Munusamy S, Saba H, Mitchell T, Megyesi JK, Brock RW, MacmillanCrow LA (2009) Alteration of renal respiratory Complex-III during experimental type-1 diabetes. BMC Endorr Disord 9: 2.

Ren S, Man RY, Angel A, Shen GX (1997) Oxidative modification enhances lipoprotein(a)-induced overproduction of plasminogen activator inhibitor-1 in cultured vascular endothelial cells. Atherosclerosis 128: $1-10$.

Ren S, Shatadal S, Shen GX (2000) Protein kinase C-beta mediates lipoprotein-induced generation of PAI-1 from vascular endothelial cells. Am J Physiol Endocrinol Metab 278: E656-E662.
Roy Chowdhury SK, Sangle GV, Xie X, Stelmack GL, Halayko AJ, Shen GX (2010) Effects of extensively oxidized low-density lipoprotein on mitochondrial function and reactive oxygen species in porcine aortic endothelial cells. Am J Physiol Endocrinol Metab 298: E89-E98.

Rustin PD, Chretien T, Bourgeron B, Gérard A, Rötig JM, Saudubray JM, Munnich A (1994) Biochemical and molecular investigations in respiratory chain deficiencies. Clin Chim Acta 228: 35-51.

Sangle GV, Chowdhury SK, Xie X, Stelmack GL, Halayko AJ, Shen GX (2010) Impairment of mitochondrial respiratory chain activity in aortic endothelial cells induced by glycated low-density lipoprotein. Free Radic Biol Med 48: 781-790.

Shen XY, Hamilton TA, Dicorleto PE (1989) Lipopolysaccharide-induced expression of the competence gene $\mathrm{KC}$ in vascular endothelial cells is mediated through protein kinase C. J Cell Physiol 140: 44-51.

Srere PA (1969) Citrate synthase. Methods Ensymol 13: 3-26.

Tsimikas S, Bergmark C, Beyer RW, Patel R, Pattison J, Miller E, Juliano J, Witztum JL (2003) Temporal increases in plasma markers of oxidized low-density lipoprotein strongly reflect the presence of acute coronary syndromes. J Am Coll Cardiol 41: 360-370.

Vondra K, Rath R, Bass A, Slabochová Z, Teisinger J, Vitek V (1977) Enzyme activities in quadriceps femoris muscle of obese diabetic male patients, Diabetologia 13: 527-529.

Zhang J, Ren S, Sun D, Shen GX (1998) Influence of glycation on LDL-induced generation of fibrinolytic regulators in vascular endothelial cells. Arterioscler Thromb Vasc Biol 18: 1140-1148.

Zhao R, Shen GX (2005) Functional modulation of antioxidant enzymes in vascular endothelial cells by glycated LDL. Atherosclerosis 179: $277-284$.

Zhao R, Ma X, Xie X, Shen GX (2009) Involvement of NADPH oxidase in oxidized LDL-induced upregulation of heat shock factor-1 and plasminogen activator inhibitor-1 in vascular endothelial cells. Am J Physiol Endocrinol Metab 297: E104-E111. 